\title{
Design Entropy Theory: A Novel Transdisciplinary Design Methodology for Smart PSS Development
}

\author{
Jingchen $\mathrm{CONG}^{\mathrm{a}, \mathrm{c}}$, Pai ZHENG ${ }^{\mathrm{b}, 1}$ and Chun-Hsien $\mathrm{CHEN}^{\mathrm{c}}$ \\ a School of Mechanical Engineering, Tianjin University, Tianjin 300350, China \\ ${ }^{b}$ Department of Industrial and Systems Engineering, Hong Kong Polytechnic \\ University, Hung Hom, Hong Kong, People's Republic of China \\ ${ }^{c}$ School of Mechanical and Aerospace Engineering, Nanyang Technological \\ University, Singapore, 639798
}

\begin{abstract}
Smart product-service systems (Smart PSS), as an emerging transdisciplinary paradigm, leverages smart, connected products (SCPs) and their generated services as a solution bundle to meet individual customer needs across different engineering fields. Owing to the advanced information and communication technologies, Smart PSS development differs from the existing product and/or service design mainly in three aspects: 1) closed-loop design/redesign iteration; 2) value co-creation in the context; and 3) design with context-awareness. These unique characteristics bring up new engineering design challenges, and to the authors' best knowledge, none of the existing design theories can address them well. Aiming to fill this gap, a novel design entroy theory is proposed by adapting the information theory with engineering design. In this context, Smart PSS can be regarded as the information container. Hence, the closed-loop design/redesign iteration can be treated as the dynamic change of information and entropy in a balanced system. Meanwhile, the value co-creation process is considered as the exchange of accumulated information via the container. Lastly, the design contextawareness represents the process of eliminating entropy. As a novel prescriptive design theory, it follows Shannon's information theory to determine the best solutions by considering the three characteristics integrally. It is envisioned this appraoch can largely facilitate today's industrial companies' digital servitization towards Industry 4.0 with better performance and user satisfaction.
\end{abstract}

Keywords. Design theory, smart product-service systems, information theory, design entropy

\section{Introduction}

In the context of industry 4.0, the current digital technologies, including Internet-ofThings (IoT), cloud/edge computing, and Big Data analytics have enabled the industrial digital transformation towards digital servitization [1]. This IT-driven value proposition paradigm is named smart product-service systems (Smart PSS) [2][3], where smart, connected products (SCPs) and their generated advanced services are delivered by the service provider/manufacturer as a solution bundle to be adopted in many disciplines.

\footnotetext{
${ }^{1}$ Corresponding Author, Mail: pai.zheng@polyu.edu.hk.
} 
Unlike other types of PSS paradigms, Smart PSS considers both the online smartness of cyberspac, and offline smartness of the physical space with sustainability concerns [4].

Nevertheless, as an emerging paradigm, none of the existing design methodologies emphasizes its multidisciplinary essence, as a sociotechnical system. Meanwhile, scarcely any work provides a fundamental approach to realize Smart PSS design by considering its transdisciplinary design characteristics (i.e., value co-creation, closedloop design, context-awareness). Aiming to fill in the gap, this research work, as the first attempt, proposes a novel design methodology for the Smart PSS development, which combines the two disciplines of engineering design and information theory. The rest of this paper is organized as follows: Section 2 presents the unique design characteristics of Smart PSS and their existing works. Section 3 provides a comprehensive review of information design and entropy in information theory. Section 4 defines design entropy theory, discusses the concept of design entropy and conversion ability, explains its design process, and introduces some formulas for the quantitative measurement of design entropy. Section 5 illustrates the concepts with examples. The conclusion and future work are summarized in Section 6 at last.

\section{Smart PSS design characteristics}

Based on the transdisciplinary nature of Smart PSS, three unique design characteristics of Smart PSS are outlined below.

Value co-creation is carried out by stakeholders who are mainly classified into three species, i.e., users, service providers, and manufacturers/vendors [5]. User participation is the most crucial part of the innovation process (e.g. user experience-driven innovation), which ensuring the real-time interaction between designers and users is fundamental to the development of Smart PSS.

Closed-loop design is conducted among SCPs and e-services, which can generate, collect, process and exchange relevant information through the system lifecycle, especially during the usage stage. Based on the useful information of the Smart PSS, the closed-loop design emphasizes the integration of innovative design and iterative design processes into the development thereby assisting the designers and engineers efficiently completing not only the creation from scratch but also the real-time upgrade/modifications.

Context-awareness is based on those intelligent systems [4]. With advanced sensors and inductive technologies provided by the intelligent systems, context-awareness can enable Smart PSS to deeply understand their customers, including their behavior, motivation and requirements. Therefore, Smart PSS involves a novel methodology which can distinguish certain contexts accurately and update solutions adaptively.

Nowadays, none of the existing methodologies which always adopted in PSS development can meet all the three characteristics. One can find that it still lacks a transdisciplinary design approach for Smart PSS. Aiming to fill this gap, a novel transdisciplinary methodology by combining two disciplines of engineering design and information theory will be proposed to solve the challenges of Smart PSS's unique characteristics in this paper. 


\section{Fundamentals of design entropy theory}

\subsection{Information design}

Information as an existence independent of material and energy [6] has a transformation relationship with data and knowledge. The researches on the information of product/service can be summarized as: (1) how to mine information as a value from usergenerated data and transfer it to users [7][8], and (2) how to manage the information generated by designers during product/service development [9][10]. The researches on the information of PSS can be basically classified into two categories, including (1) the concept of information modules can be proposed and used as a core module [11][12] to replace the product and service modules [13], and (2) an information system can be established to record and update the input and output information of each module under different contexts for completing and selecting the solutions more accurately [14].

\subsection{Entropy}

Shannon (1948) [15] systematically discussed the fundamental issues of information communication by using mathematical tools (e.g., probability theory and statistics) to propose the quantitative expression of information, the calculation of transmission rate, the calculation of channel capacity, and the coding theorems of information transmission. Menhorn et al. (2011) [16] presented design entropy as a measurement for the complexity of a given circuit by resorting to Shannon's information theory, which is mainly used in the digital circuits field rather than the design field. Based on the entropy in information theory, Wu et al. (2016) [17] defined design entropy as a description of the disorder found in design objects and proposed a design entropy model, which is a measurement of the degree of information chaos in a user interface. Since most design problems of Smart PSS need to extract and summarize information in the context, which cannot be solved effeciently by the existing design entropy methods.

It should be noted that, Axiomatic Design also adopts the information theory [18] to determine the probability distribution of the system range and the common range to compute the system information content. Nevertheless, it only considers the mapping process of the pre-defined product domains for solution evalution. Both the in-context product-service information and the conversion capabilities are ignored.

\section{Design Entropy Theory}

\subsection{Introduction of Design entropy theory}

Due to the intelligentization, digitization and servitization characteristics of Smart PSS, it should take information stored and transmitted in the system as the research objective, instead of physical substance. As depicted in Figure 1, when the Smart PSS reaches the end of lifecycle, the physical substance will be disposed, and only its parts and materials can be reused and recycled. However, information which largely differs from the physical substances can be transmitted to the new Smart PSS completely.

From an informational perspective, design entropy theory regards Smart PSS as the carriers of information, and the design process as the conversion from the information to physical products or services. It is favorable to the establishment of a Smart PSS 
ecosystem by considering a series of interconnected systems, not a merely single system. The information in smart PSS can be input by designers, engineers, and other stakeholders directly. Meanwhile, it also can be extracted from the environment data collected by SCP sensors, the behavior data generated by customers in using period, and the online data of social networks.

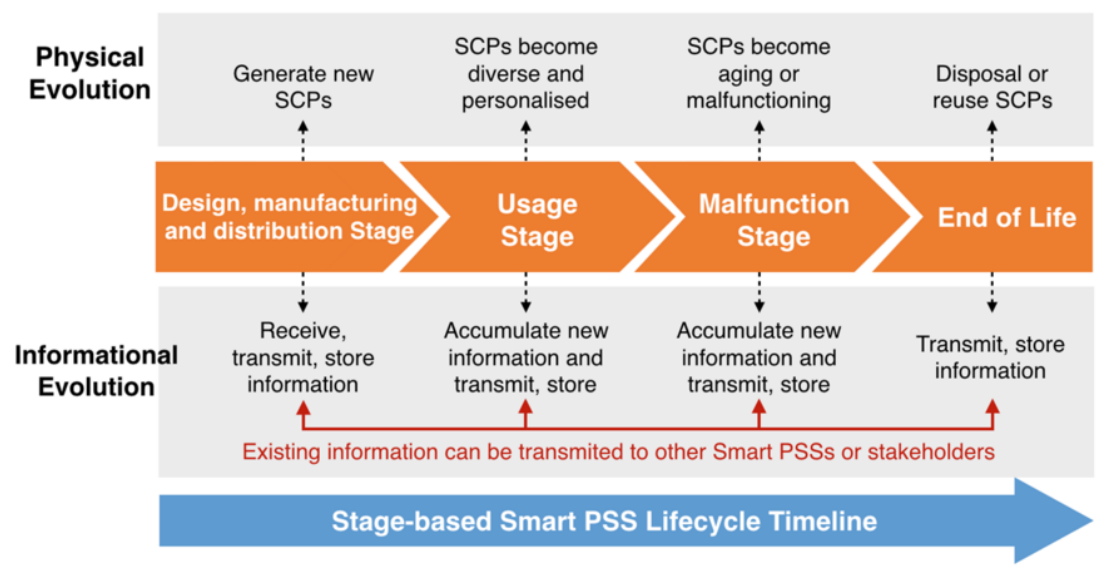

Figure 1. Physical and Informatic evolution in Smart PSS lifecycle.

\subsection{Design entropy}

Before proposing the definition of design entropy, the scope of information in Smart PSS needs to be explained. Information in Smart PSS is defined as the descriptive information which describes the status of the stakeholders, SCPs, services, and environment, and introduces the solutions. Meanwhile, design plan of Smart PSS differs from the traditional nomenclature, which is a description (blueprint or CAD model) of the Smart PSS. It mainly consists of three parts, including the SCP design plan (usually displayed as a CAD model), service design plan (usually displayed as a service blueprint), and conversion plan that will be mentioned in Section 4.5.

Design entropy could be a measure of information design in Smart PSS for describing its certainty degree. The absolute value of design entropy is positively correlated with the uncertainty of systems. If system certainty is higher, it will cause design entropy to be lower, the accuracy of its context awareness to be higher, quality of real-time iterative design based on the current context to be better, and user participation and satisfaction degree to be higher. Therefore, Design entropy theory needs to dynamically reduce the design entropy for maintaining the highest certainty of the system and ensuring the most exceptional adaptability for each context during usage stage.

To calculating design entropy, we define a new measure called Conversion ability, which is denoted by $\mathrm{C}$. Conversion ability represents the ability to convert the information, which is denoted by $x_{i}$ into the information, which is denoted by $x^{\prime}$. If the conversion ability of Smart PSS is higher, its conversion effect from $x$ to $x^{\prime}$ to be better and design entropy of this system to be lower.

Let the design entropy of a system be represented by $D$; the value of $D$ is evaluated:

$$
D=-\log C
$$


, where $0 \leq C \leq 1$, and all $\mathrm{D} \geq 0$. Thus if the conversion ability of a system is the highest, the value of $C$ is 1 , then $\mathrm{D}=0$. On the contrary, the value of $\mathrm{C}$ is 0 , then $\mathrm{D}=$ $+\infty$. In order to compare the value of design entropy in different contexts and systems, the same logarithmic base 2 is used in each equation of design entropy. Furthermore, the total design entropy of a system is given by

$$
D_{\text {total }}=D_{\text {innovative }}+D_{\text {iterative }}
$$

, where $D_{\text {innovative }}$ is innovative design entropy of the system, and $D_{\text {iterative }}$ is iterative design entropy. The former one means the design entropy in the innovative design stage of creating a new Smart PSS. Moreover, the latter one means the design entropy in the iterative design stage, when redesigning, modifying, or enhancing the solutions according to the new information collected from sensors during use.

\subsection{Innovative design entropy and innovative conversion ability}

The innovative design entropy of a system is defined as $D_{\text {innovative }}$, according to the innovative conversion of the system, $C_{\text {innovative }}$ :

$$
D_{\text {innovative }}=-\log C_{\text {innovative }}
$$

This quantity measures how uncertain we are of the system in its innovative design stage when we know $C_{\text {innovative }}$. $C_{\text {innovative }}$ refers to the conversion ability in the design plan so that this design can effectively convert information in usage stages. $C_{\text {innovative }}$ is further denoted as:

$$
C_{\text {innovative }}=A_{1} \times Y_{1}+A_{2} \times Y_{2}+\cdots+A_{n} \times Y_{n}
$$

, where $Y_{n}$ is a parameter that represents the ability that, design plan can convert information in a specific way when the design is used in the future. Since $0 \leq \mathrm{C} \leq 1$, we let $0 \leq Y_{n} \leq 1$. Different research objects have different parameters. The matching degree with user requirements, adaptability measure, and the number of sensors are utilized according to the unique characteristics of Smart PSS proposed. After the normalization processing, these three parameters are used as $Y_{1}, Y_{2}, Y_{3}$. Furthermore, coefficient $A_{\mathrm{n}}$ is the weighting factor introduced because the importance of $Y_{n}$ is different in each case. $A_{\mathrm{n}}$ is given by the stakeholders for expressing the importance of $\mathrm{Y}_{\mathrm{n}}$ to the system. Thus $0 \leq C_{\text {innovative }} \leq 1$ and $0 \leq Y_{n} \leq 1$, where

$$
A_{1}+A_{2}+\cdots+A_{n}=1
$$

\subsection{Iterative design entropy and iterative conversion ability}

Figure 2 illustrates the process of information conversion in the usage stage of Smart PSS. After collecting new information, Smart PSS builds and updates the usage context according to this novel one. In a specific usage scenario, Smart PSS should firstly determine whether the information is noise, which refers to the useless information for design in the current context, and delete it if the information is noise. If it is not noise, this information $x$ should convert into the information $x^{\prime}$. After this processing step, the information should be stored or deleted. 


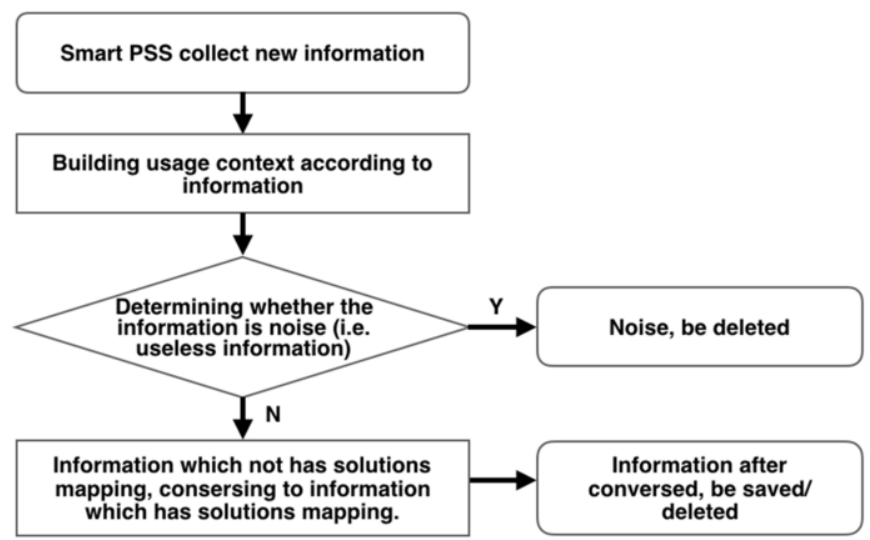

Figure 2. Process of information conversion in usage stage.

Iterative design entropy consists of the sum of the design entropy of all non-noisy and unconverted information $x$, which is collected by the system during the use phase as:

$$
D_{\text {iterative }}=\sum_{x \in \mathcal{X}} D(x)
$$

, where $\mathcal{X}$ is the set of all non-noisy and unconverted information collected by the system, and information $x$ belongs to the set $\mathcal{X}$. The information $x_{i}$ converted into $m$ pieces of information $x^{\prime}$ (i.e., $x_{1}{ }^{\prime}$ to $x_{m}{ }^{\prime}$ ), through $m$ items of channels. The design entropy of information $x_{i}$ is

$$
D\left(x_{i}\right)=-\sum_{m \in \mathcal{M}} W\left(x_{i}\right)_{m} \log C\left(x_{i}\right)_{m}
$$

, where $W\left(x_{i}\right)_{m}$ is the weighting factor of channel $m$, representing the correlation of information $x_{i}$ and $x_{m}{ }^{\prime} . W\left(x_{i}\right)_{m}$, which is modified in a diverse context, is given by the stakeholders. $\mathcal{M}$ is the set of all channels between information $x_{i}$ and information after conversion, and channel $m$ belongs to the set $\mathcal{M}$. $C\left(x_{i}\right)_{m}$ refers to the conversion ability to convert information $x_{i}$ into $x_{m}{ }^{\prime}$ through channel $m$, representing the singlechannel conversion capability for a single piece of information in the system.

$C\left(x_{i}\right)_{\mathrm{m}}=A\left(x_{i}\right)_{\mathrm{m}_{1}} \times Y\left(x_{i}\right)_{\mathrm{m}_{1}}+A\left(x_{i}\right)_{\mathrm{m}_{2}} \times Y\left(x_{i}\right)_{\mathrm{m}_{2}}+\cdots+A\left(x_{i}\right)_{\mathrm{m}_{n}} \times Y\left(x_{i}\right)_{\mathrm{m}_{n}}$

, where $Y\left(x_{i}\right)_{\mathrm{m}_{n}}$ is a parameter that represents the ability that, information $x_{i}$ can convert into information $x_{m}{ }^{\prime}$ in a specific way efficiently and accurately. Different information conversion types have different parameters. The user satisfaction degree, time and cost of completing information conversion are utilized according to the unique characteristics of Smart PSS proposed. After the normalization processing, these three parameters are used as $Y\left(x_{i}\right)_{\mathrm{m}_{1}}, Y\left(x_{i}\right)_{\mathrm{m}_{2}}, Y\left(x_{i}\right)_{\mathrm{m}_{3}}$. Coefficient $A(x)_{\mathrm{m}_{n}}$, which is given by the stakeholders, refers to the weighting factor of the importance of $Y\left(x_{i}\right)_{\mathrm{m}_{n}}$.

\subsection{Design process of Design entropy theory}

Figure 3 shows the design process by using design entropy theory. In the innovative design phase, a design plan needs to be proposed, and the innovative design entropy of the plan need to be calculated. The entropy should be reduced as possible, and the plan with the lowest design entropy should be selected. In the conversion plan, it is necessary 
to predict and list information $x$, which will be collected in future stages, and design its conversion plan and converted information $x^{\prime}$. After manufacturing, Smart PSS will be used by customers. Collecting new information continuously during the usage stage makes iterative design entropy increase. Therefore, Smart PSS needs to convert the information in real-time to reduce the design entropy.

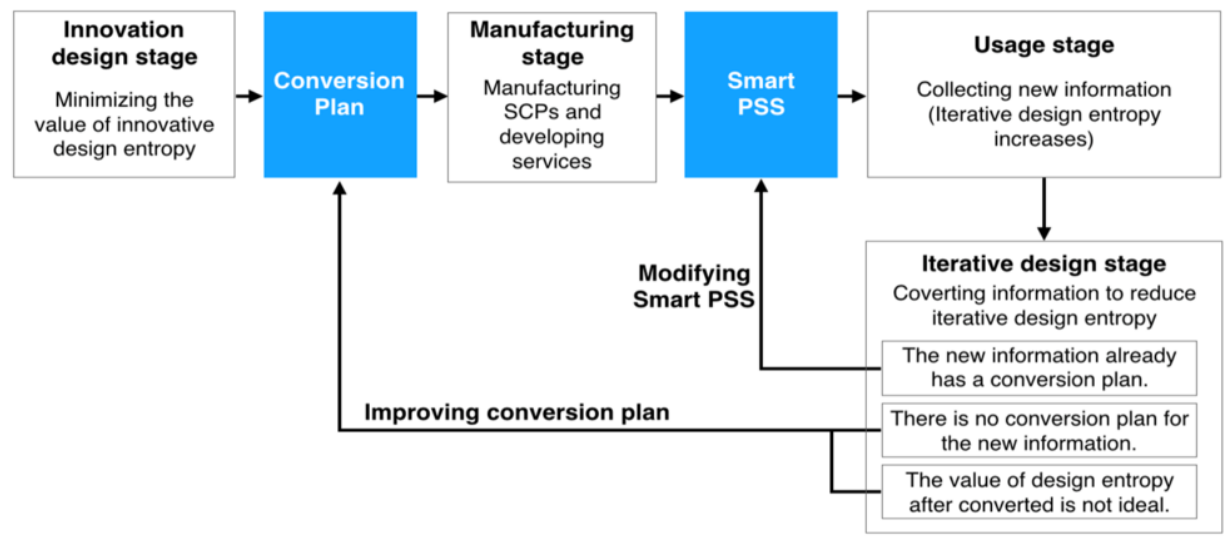

Figure 3. Design process of design entropy theory.

\section{Case Study}

Empirical case studies have proved as effective model verification methods [7][23]. Hereby, this section provides a case study of smart travel assistant product-service system to verify the effectiveness of the proposed design entropy theory. Smart assistants (e.g. service robot, navigation APP) have been increasingly introduced in recent years. Elderly users always live in the local retirement homes, so that they need it to address their traveling requirements, such as: sending the health status of elderly to their relatives in real-time, and assisting elderly in choosing and visiting the tourism destination.

Step 1: Select the design plan with the lowest innovative design entropy. Figure 4 shows the prototypes of the smart travel assistant design plans, and their innovative design entropy. After calculating and comparing, plan 1 with the lowest innovative design entropy should be selected for subsequent development.

Step 2: Predict and list the information to be collected in different usage contexts. As shown in Figure 4, the information of the smart travel assistant is summarized from three primary contexts of the elderly users' usage.

\section{Step 3: Planning the information $x_{m}{ }^{\prime}$ after conversion.}

Figure 4 shows the preliminary conversion plan of the smart travel assistant. At this stage, the developers should analyze information $x_{i}$ holistically and give the corresponding information $\mathrm{x}_{\mathrm{m}}{ }^{\prime}$ after conversion. The developers need to conceive of the conversion plan by combining the design plan and the requirements of stakeholders.

Step 4: Mark weighting factor $W\left(x_{i}\right)_{m}$ and output information conversion plan. Figure 4 shows the information conversion plan of the smart travel assistant. The conversion plan should be completed before SCPs manufacture and service development. The conversion plan will be continuously adapted and iterated during usage. 


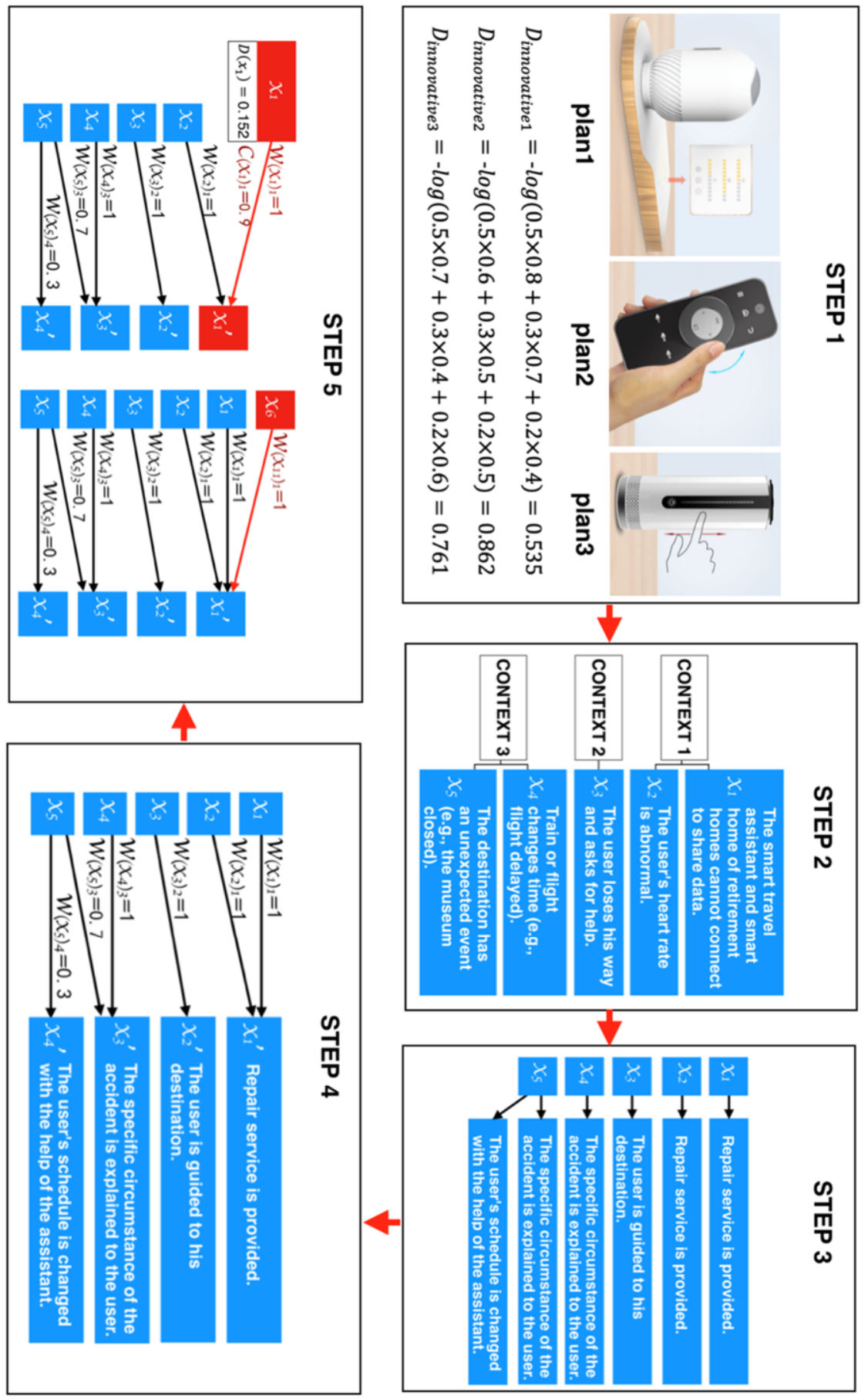

Figure 4. Design process of the smart travel assistant with design entropy theory. 
Step 5: Execute or iterate the conversion plan during customers usage phase. The value of iterative design entropy needs to be monitored in real-time during the usage stage to enable that the value is as expected:1) If the newly collected information was predicted in a conversion plan before, the conversion channel of this information can be activated directly, and the system should provide services, adjust parameters, or replace modules according to the corresponding information $x_{m}{ }^{\prime}$. The conversion ability can be calculated and recorded by obtaining the user satisfaction degree, time, and cost of completing information conversion (Figure 4). 2) If the newly collected information has not been predicted before, the design entropy of this information will tend to infinity. The system should modify the conversion plan, including converting the information $x_{6}$ ' to an existing information $x_{1}{ }^{\prime}$, or converting it to a new information $x_{6}{ }^{\prime}$.

\section{Conclusion and future works}

Smart PSS, as an emerging IT-driven transdisciplinary paradigm coined in 2014, has attracted ever-increasing attention among academics recently. Nevertheless, there still lacks a fundamental design methodology for Smart PSS development. To bridge this gap, this paper presents a novel design entropy approach that focuses on developing Smart PSS by combining the two disciplines of engineering design and information theory. It depicts the fundamental mechanism of the design literation process in the Smart PSS. Moreover, it also provides insightful potentials for complex sociotechnical systems to quickly satisfy customer requirements in a context-aware manner. As an explorative theorectical study, future work can also be done in the following aspects:

1) Automatically monitoring the value of the design entropy. Automatic supervision will help designers only modify the plan when the design entropy does not meet the standards, instead of keeping observing the value of design entropy, which could contribute to higher productivity through transdisciplinary engineering.

2) Building a general platform for conversion plan: A general platform for conversion plan can be built to collect conversion plans from different Smart PSS, which need intensive collaboration across different disciplines.

It is envisioned this appraoch can largely facilitate today's industrial companies' digital servitization towards Industry 4.0 with better performance and user satisfaction.

\section{References}

[1] C. Lerch and M. Gotsch, Digitalized Product-Service Systems in Manufacturing Firms: A Case Study Analysis, Research-Technology Management, vol. 58, no. 5, pp. 45-52, Sep. 2015, doi: 10.5437/08956308X5805357.

[2] A. Valencia, R. Mugge, J. P. L. Schoormans, and H. N. J. Schifferstein, Challenges in the design of smart product-service systems (PSSs): Experiences from practitioners, in 19th DMI Academic Design Management Conference, no. September, pp. 1-21, 2014.

[3] P. Zheng, T.-J. Lin, C.-H. Chen, and X. Xu, A systematic design approach for service innovation of smart product-service systems, Journal of Cleaner Production, vol. 201, pp. 657-667, Nov. 2018, doi: 10.1016/j.jclepro.2018.08.101. 
[4] P. Zheng, Z. Wang, C.-H. Chen, and L. Pheng Khoo, A survey of smart productservice systems: Key aspects, challenges and future perspectives, Advanced Engineering Informatics, Oct. 2019, vol. 42, p. 100973, doi: 10.1016/j.aei.2019.100973.

[5] P. Zheng, Z. Wang, and C.-H. Chen, Industrial smart product-service systems solution design via hybrid concerns, Procedia CIRP, 2019, vol. 65, pp. 32-37, doi: 10.1016/j.procir.2017.04.009.

[6] N. Wiener, Cybernetics: Or Control and Communication in the Animal and the Machine, MIT Press, Boston, 1948.

[7] C.-H. Lim, M.-J. Kim, J.-Y. Heo and K.-J. Kim, Design of informatics-based services in manufacturing industries: case studies using large vehicle-related databases, Journal of Intelligent Manufacturing, Mar. 2015, vol. 29, no. 3, pp. 497-508, doi: 10.1007/s10845-015-1123-8.

[8] C. Lim, K.-H. Kim, M.-J. Kim, J.-Y. Heo, K.-J. Kim, and P. P. Maglio, From data to value: A nine-factor framework for data-based value creation in informationintensive services, International Journal of Information Management, Apr. 2018, vol. 39, pp. 121-135, doi: 10.1016/j.ijinfomgt.2017.12.007.

[9] C. McMahon, Design Informatics: Supporting Engineering Design Processes with Information Technology, Journal of the Indian Institute of Science, 2015, Vol. 95, pp. 365-377.

[10] A. J. Wodehouse and W. J. Ion, Information use in conceptual design: existing taxonomies and new approaches, International Journal of Design, 2010, vol. 4, pp. 53-65.

[11] D. Opresnik and M. Taisch, The value of Big Data in servitization, International Journal of Production Economics, Jul. 2015, vol. 165, pp. 174-184, doi: 10.1016/j.ijpe.2014.12.036.

[12] T. Baines and H. W. Lightfoot, Servitization of the manufacturing firm: Exploring the operations practices and technologies that deliver advanced services, International Journal of Operations \& Production Management, Dec. 2013, vol. 34, no. 1, pp. 2-35, doi: 10.1108/IJOPM-02-2012-0086.

[13] J. Cenamor, D. Rönnberg Sjödin, and V. Parida, Adopting a platform approach in servitization: Leveraging the value of digitalization, International Journal of Production Economics, Oct. 2017, vol. 192, pp. 54-65, doi: 10.1016/j.ijpe.2016.12.033.

[14] J. C. Aurich, C. Fuchs, and C. Wagenknecht, Life cycle oriented design of technical Product-Service Systems, Journal of Cleaner Production, Jan. 2006, vol. 14, no. 17, pp. 1480-1494, doi: 10.1016/j.jclepro.2006.01.019.

[15] C. E. Shannon, A Mathematical Theory of Communication, 1949, http://people.math.harvard.edu/ ctm/home/text/others/shannon/entropy/entropy. pdf, accessed July, 12020.

[16] B. Menhorn and F. Slomka, Design entropy concept: a measurement for complexity, in Proceedings of the seventh IEEE/ACM/IFIP international conference on Hardware/software codesign and system synthesis CODES+ISSS '11, Taipei, Taiwan, 2011, p. 285, doi: 10.1145/2039370.2039414.

[17] L. Wu, J. Li, and T. Lei, Design Entropy: A New Approach for Evaluating User Experience in User Interface Design, in Advances in Ergonomics in Design, vol. 485, F. Rebelo and M. Soares, Eds. Cham: Springer International Publishing, 2016, pp. 583-593.

[18] N. P. Suh, The Principles of Design, Oxford University Press, Oxford, 1990. 